International Mathematical Forum, Vol. 8, 2013, no. 34, 1691 - 1702

HIKARI Ltd, www.m-hikari.com

http://dx.doi.org/10.12988/imf.2013.39176

\title{
Multiparameter K-Mittag-Leffler Function
}

\author{
Kuldeep Singh Gehlot \\ Government Bangur P.G. College, Pali \\ Pali-Marwar, Rajasthan, India-306401 \\ drksgehlot@rediffmail.com
}

Copyright (C) 2013 Kuldeep Singh Gehlot. This is an open access article distributed under the Creative Commons Attribution License, which permits unrestricted use, distribution, and reproduction in any medium, provided the original work is properly cited.

\begin{abstract}
In this paper author introduce Multiparameter K-Mittag-Leffler Function definded as,

$$
\begin{gathered}
{ }_{p} K_{q, k}^{(\beta, \eta)_{m}}[z]={ }_{p} K_{q, k}^{(\beta, \eta)_{m}}\left[a_{1}, . ., a_{p} ; b_{1}, . ., b_{q},\left(\beta_{1}, \eta_{1}\right), . .,\left(\beta_{m}, \eta_{m}\right) ; z\right], \\
{ }_{p} K_{q, k}^{(\beta, \eta)_{m}}[z]=\sum_{n=0}^{\infty} \frac{\prod_{j=1}^{p}\left(a_{j}\right)_{n, k} z^{n}}{\prod_{r=1}^{q}\left(b_{r}\right)_{n, k} \prod_{i=1}^{m} \Gamma_{k}\left(\eta_{i} n+\beta_{i}\right)},
\end{gathered}
$$

where $k \in R_{+}=(0, \infty) ; a_{j}, b_{r}, \beta_{i} \in C ; \eta_{i} \in R(j=1,2, . ., p ; r=1,2, . ., q ; i=$ $1,2, . ., m)$.

Certain relations that exist between ${ }_{p} K_{q, k}^{(\beta, \eta)_{m}}[z]$ function and Riemann-Liouville fractional integral and derivatives has been evaluated. It has been shown that the fractional integration and differentation of ${ }_{p} K_{q, k}^{(\beta, \eta)_{m}}[z]$ function with power multipliers into the function of the same form.

Also deduce Mittag-Leffler functions introduced by [1],[3],[4],[5],[6],[7],[8],[13],,[14],,[15] and [16] are particular cases of Multiparameter K-Mittag-Leffler function for some particular values of parameters and deduce some particular cases.
\end{abstract}

Mathematics Subject Classification: 26A33, 33E12, 33C20

Keywords: Multiparameter K-Mittag-Leffler function, K-Series, K-Pochhammer symbol, K- Gamma function, Riemann-Liouville fractional operators 


\section{Introduction}

In [9] the author introduce the generalized K-Gamma Function $\Gamma_{k}(x)$ as

$$
\Gamma_{k}(x)=\lim _{n \rightarrow \infty} \frac{n ! k^{n}(n k)^{\frac{x}{k}-1}}{(x)_{n, k}}, k>0, x \in C \backslash k Z^{-},
$$

where $(x)_{n, k}$ is the $\mathrm{k}$-Pochhammer symbol and is given by

$$
(x)_{n, k}=x(x+k)(x+2 k) \ldots . .(x+(n-1) k), x \in C, k \in R, n \in N^{+} .
$$

$\mathrm{K}$-Gamma function is given by,

$$
\Gamma_{k}(x)=\int_{0}^{\infty} t^{x-1} e^{-\frac{t^{k}}{k}} d t, x \in C, k \in R, \operatorname{Re}(x)>0,
$$

and it follows easily that

$$
\Gamma_{k}(x)=k^{\frac{x}{k}-1} \Gamma\left(\frac{x}{k}\right) \text { and }(\gamma)_{n q, k}=(k)^{n q}\left(\frac{\gamma}{k}\right)_{n q} .
$$

The Fractional Integral operators for $\alpha>0$ ([10], Definition 2.1, Page 33) are defined as,

$$
\begin{aligned}
& \left(I_{0+}^{\alpha} f\right)(x)=\frac{1}{\Gamma(\alpha)} \int_{0}^{x} \frac{f(t)}{(x-t)^{1-\alpha}} d t,(x>0), \\
& \left(I_{-}^{\alpha} f\right)(x)=\frac{1}{\Gamma(\alpha)} \int_{x}^{\infty} \frac{f(t)}{(t-x)^{1-\alpha}} d t,(x>0),
\end{aligned}
$$

The Fractional Derivative for $\alpha>0$ ([10], Definition 2.2, Page 35) are defined as,

$$
\begin{aligned}
& \left(D_{0+}^{\alpha} f\right)(x)=\left(\frac{d}{d x}\right)^{[\alpha]+1}\left(I_{0+}^{1-\{\alpha\}} f\right)(x)=\frac{1}{\Gamma(1-\{\alpha\})}\left(\frac{d}{d x}\right)^{[\alpha]+1} \int_{0}^{x} \frac{f(t)}{(x-t)^{\{\alpha\}}} d t,(x>0), \\
& \left(D_{-}^{\alpha} f\right)(x)=\left(\frac{d}{d x}\right)^{[\alpha]+1}\left(I_{-}^{1-\{\alpha\}} f\right)(x)=\frac{1}{\Gamma(1-\{\alpha\})}\left(-\frac{d}{d x}\right)^{[\alpha]+1} \int_{x}^{\infty} \frac{f(t)}{(t-x)^{\{\alpha\}}} d t,(x>0) .
\end{aligned}
$$

where $[\alpha]$ means the maximal integer not extending $\alpha$ and $\{\alpha\}$ is the fractional part of $\alpha$.

The next assertion is well known; see ([10], (2.44) and table 9.3, formula 1). Let $\alpha \in C,(\operatorname{Re}(\alpha)>0)$ and $\gamma \in C$.

$$
\begin{gathered}
\text { if } \operatorname{Re}(\gamma)>0, \text { then }\left(I_{0+}^{\alpha} t^{\gamma-1}\right)(x)=\frac{\Gamma(\gamma)}{\Gamma(\alpha+\gamma)} x^{\alpha+\gamma-1} \\
\text { if } \operatorname{Re}(\gamma)>\operatorname{Re}(\alpha)>0, \operatorname{then}\left(I_{-}^{\alpha} t^{-\gamma}\right)(x)=\frac{\Gamma(\gamma-\alpha)}{\Gamma(\gamma)} x^{\alpha-\gamma}
\end{gathered}
$$




\section{Main result}

In this section we introduce Multiparameter K-Mittag-Leffler function and for particular values of parameter we can deduce Multiparameter K-Mittag-Leffler function in to eariler known Mittag-Leffler functions. Finally we calculate Fractional integral and derivative of Multiparameter K-Mittag-Leffler function.

\subsection{Multiparameter K-Mittag-Leffer Function}

Definition: Let $k \in R_{+}=(0, \infty) ; a_{j}, b_{r}, \beta_{i} \in C ; \eta_{i} \in R(j=1,2, . ., p ; r=$ $1,2, . ., q ; i=1,2, . ., m)$. Then the Multiparameter K-Mittag-Leffler function definded as,

$$
{ }_{p} K_{q, k}^{(\beta, \eta)_{m}}\left[a_{1}, . ., a_{p} ; b_{1}, . ., b_{q},\left(\beta_{1}, \eta_{1}\right), . .,\left(\beta_{m}, \eta_{m}\right) ; z\right]=\sum_{n=0}^{\infty} \frac{\prod_{j=1}^{p}\left(a_{j}\right)_{n, k} z^{n}}{\prod_{r=1}^{q}\left(b_{r}\right)_{n, k} \prod_{i=1}^{m} \Gamma_{k}\left(\eta_{i} n+\beta_{i}\right)}
$$

Where $\Gamma_{k}(x)$ is the K-Gamma function given by $(1)$ and $(\gamma)_{n, k}$ is the KPochhammer symbol given by (2).

The series (11) is definded when none of the parameter $b_{r}(r=1,2, . ., q)$ is negative integer or zero. If any parameter $a_{j}(j=1,2, . ., p)$ in $(11)$ is zero or negative, the series terminates into polynomial in $\mathrm{z}$.

Convergent conditions for the series (11) are given by Ratio test,

(i) If $p<q+\sum_{i=1}^{m}\left(\frac{\eta_{i}}{k}\right)$, then the power series on the right of (11) is absolutely convergent for all $z \in C$.

(ii) If $p=q+\sum_{i=1}^{m}\left(\frac{\eta_{i}}{k}\right)$, then the power series on the right of (11) is absolutely convergent for all $\left|k^{p-q-\sum_{i=1}^{m}\left(\frac{\eta_{i}}{k}\right)} z\right|<\prod_{i=1}^{m}\left(\left|\frac{\eta_{i}}{k}\right|\right)^{\frac{\eta_{i}}{k}}$ and $\left|k^{p-q-\sum_{i=1}^{m}\left(\frac{\eta_{i}}{k}\right)} z\right|=$ $\prod_{i=1}^{m}\left(\left|\frac{\eta_{i}}{k}\right|\right)^{\frac{\eta_{i}}{k}}, \operatorname{Re}\left(\sum_{r=1}^{q}\left(\frac{b_{r}}{k}\right)+\sum_{i=1}^{m}\left(\frac{\beta_{i}}{k}\right)-\sum_{j=1}^{p}\left(\frac{a_{j}}{k}\right)\right)>\frac{2+q+m-p}{2}$.

\subsubsection{Particular cases}

By particularizing the parameters in (11), we obtain following known Mittagleffler functions.

(a) If we set $k=1$ in (11), we have

$$
\begin{gathered}
{ }_{p} K_{q, 1}^{(\beta, \eta)_{m}}\left[a_{1}, . ., a_{p} ; b_{1}, . ., b_{q},\left(\beta_{1}, \eta_{1}\right), . .,\left(\beta_{m}, \eta_{m}\right) ; z\right]=\sum_{n=0}^{\infty} \frac{\prod_{j=1}^{p}\left(a_{j}\right)_{n} z^{n}}{\prod_{r=1}^{q}\left(b_{r}\right)_{n} \prod_{i=1}^{m} \Gamma\left(\eta_{i} n+\beta_{i}\right)}, \\
{ }_{p} K_{q, 1}^{(\beta, \eta)_{m}}\left[a_{1}, . ., a_{p} ; b_{1}, . ., b_{q},\left(\beta_{1}, \eta_{1}\right), . .,\left(\beta_{m}, \eta_{m}\right) ; z\right]={ }_{p} K_{q}^{(\beta, \eta)_{m}}[z] .
\end{gathered}
$$


Which is K-Series definded by [3,4].

(b) If we set $k=1, p=q=m$ and $b_{1}=b_{2}=\ldots=b_{m}=1$ in (11), we have

$$
\begin{gathered}
{ }_{m} K_{m, 1}^{(\beta, \eta)_{m}}\left[a_{1}, . ., a_{m} ; 1,1, . ., 1,\left(\beta_{1}, \eta_{1}\right), . .,\left(\beta_{m}, \eta_{m}\right) ; z\right]=\sum_{n=0}^{\infty} \frac{\prod_{i=1}^{m}\left(a_{i}\right)_{n} z^{n}}{\prod_{i=1}^{m} \Gamma\left(\eta_{i} n+\beta_{i}\right)(n !)^{m}}, \\
{ }_{m} K_{m, 1}^{(\beta, \eta)_{m}}\left[a_{1}, . ., a_{m} ; 1,1, . ., 1,\left(\beta_{1}, \eta_{1}\right), . .,\left(\beta_{m}, \eta_{m}\right) ; z\right]=E_{\left(\eta_{i}\right),\left(\beta_{i}\right)}^{\left(a_{i}\right), m}[z] .
\end{gathered}
$$

Which is the 3M-Parameter Multi-Index Mittag-Leffler function definded by $[5]$.

(c) If we set $k=1, p=q=1, a_{1}=\rho, b_{1}=1$ in (11), then we obtain

$$
\begin{gathered}
{ }_{1} K_{1,1}^{(\beta, \eta)_{m}}\left[\rho ; 1,\left(\beta_{1}, \eta_{1}\right), . .,\left(\beta_{m}, \eta_{m}\right) ; z\right]=\sum_{n=0}^{\infty} \frac{(\rho)_{n} z^{n}}{\prod_{i=1}^{m} \Gamma\left(\eta_{i} n+\beta_{i}\right)(n !)} \\
{ }_{1} K_{1,1}^{(\beta, \eta)_{m}}\left[\rho ; 1,\left(\beta_{1}, \eta_{1}\right), . .,\left(\beta_{m}, \eta_{m}\right) ; z\right]=E_{\rho}\left[(\eta, \beta)_{m} ; z\right] .
\end{gathered}
$$

Which is the Generalized Mittag-Leffler function studied by [6].

(d) If we set $k=1, p=q=1, a_{1}=b_{1}=1$ and $\eta_{i}=\frac{1}{\alpha_{i}}$ in (11), then we obtain

$$
\begin{gathered}
{ }_{1} K_{1,1}^{(\beta, \eta)_{m}}\left[1 ; 1,\left(\beta_{1}, \frac{1}{\alpha_{1}}\right), . .,\left(\beta_{m}, \frac{1}{\alpha_{m}}\right) ; z\right]=\sum_{n=0}^{\infty} \frac{z^{n}}{\prod_{i=1}^{m} \Gamma\left(n \frac{1}{\alpha_{i}}+\beta_{i}\right)}, \\
{ }_{1} K_{1,1}^{(\beta, \eta)_{m}}\left[1 ; 1,\left(\beta_{1}, \frac{1}{\alpha_{1}}\right), . .,\left(\beta_{m}, \frac{1}{\alpha_{m}}\right) ; z\right]=E_{\left(\frac{1}{\alpha_{i}}\right),\left(\beta_{i}\right)}^{(m)}[z] .
\end{gathered}
$$

Which is the Multi-Index Mittag-Leffler function studied by [15].

(e) If we set $k=1, m=1$ in (11), we have

$$
\begin{aligned}
& { }_{p} K_{q, 1}^{(\beta, \eta)_{1}}\left[a_{1}, . ., a_{p} ; b_{1}, . ., b_{q},(\beta, \eta) ; z\right]=\sum_{n=0}^{\infty} \frac{\prod_{j=1}^{p}\left(a_{j}\right)_{n} z^{n}}{\prod_{r=1}^{q}\left(b_{r}\right)_{n} \Gamma(\eta n+\beta)} . \\
& { }_{p} K_{q, 1}^{(\beta, \eta)_{1}}\left[a_{1}, . ., a_{p} ; b_{1}, . ., b_{q},(\beta, \eta) ; z\right]={ }_{p} M_{q}^{\eta, \beta}\left[a_{1}, . ., a_{p} ; b_{1}, . ., b_{q} ; z\right] .
\end{aligned}
$$

Which is Generalized M-Series definded by [13].

(f) If we set $k=1, m=1$ and $\beta=1$ in (11), we have

$$
{ }_{p} K_{q, 1}^{(\beta, \eta)_{1}}\left[a_{1}, . ., a_{p} ; b_{1}, . ., b_{q},(1, \eta) ; z\right]=\sum_{n=0}^{\infty} \frac{\prod_{j=1}^{p}\left(a_{j}\right)_{n} z^{n}}{\prod_{r=1}^{q}\left(b_{r}\right)_{n} \Gamma(\eta n+1)} .
$$




$$
{ }_{p} K_{q, 1}^{(\beta, \eta)_{1}}\left[a_{1}, . ., a_{p} ; b_{1}, . ., b_{q},(1, \eta) ; z\right]={ }_{p} M_{q}^{\eta}\left[a_{1}, . ., a_{p} ; b_{1}, . ., b_{q} ; z\right] .
$$

Which is M-Series definded by [14].

(g) If we set $p=q=m=1, a_{1}=\delta, b_{1}=k$ in (11),then we obtain

$$
\begin{gathered}
{ }_{1} K_{1,1}^{(\beta, \eta)_{1}}[\delta ; k,(\beta, \eta) ; z]=\sum_{n=0}^{\infty} \frac{(\delta)_{n, k}}{\Gamma_{k}(\eta n+\beta)(n !)}\left(\frac{z}{k}\right)^{n} \\
{ }_{1} K_{1,1}^{(\beta, \eta)_{1}}[\delta ; k,(\beta, \eta) ; z]=E_{k, \eta, \beta}^{\delta}\left[\frac{z}{k}\right] .
\end{gathered}
$$

Which is the K- Mittag-Leffler function studied by [1].

(h) If we set $k=1, p=q=m=1, a_{1}=\delta, b_{1}=1$ in (11), then we obtain

$$
\begin{gathered}
{ }_{1} K_{1,1}^{(\beta, \eta)_{1}}[\delta ; 1,(\beta, \eta) ; z]=\sum_{n=0}^{\infty} \frac{(\delta)_{n} z^{n}}{\Gamma(\eta n+\beta)(n !)}, \\
{ }_{1} K_{1,1}^{(\beta, \eta)_{1}}[\delta ; 1,(\beta, \eta) ; z]=E_{\eta, \beta}^{\delta}[z] .
\end{gathered}
$$

Which is the Generalized Mittag-Leffler function studied by [8].

(i) If we set $k=1, p=q=m=1, a_{1}=b_{1}=1$ in (11), then we obtain

$$
\begin{gathered}
{ }_{1} K_{1,1}^{(\beta, \eta)_{1}}[1 ; 1,(\beta, \eta) ; z]=\sum_{n=0}^{\infty} \frac{z^{n}}{\Gamma(\eta n+\beta)}, \\
{ }_{1} K_{1,1}^{(\beta, \eta)_{1}}[1 ; 1,(\beta, \eta) ; z]=E_{\eta, \beta}[z] .
\end{gathered}
$$

Which is the Mittag-Leffler function studied by [16].

(j) If we set $k=1, p=q=m=1, a_{1}=b_{1}=1$ and $\beta=1$ in (11),then we obtain

$$
\begin{gathered}
{ }_{1} K_{1,1}^{(\beta, \eta)_{1}}[1 ; 1,(1, \eta) ; z]=\sum_{n=0}^{\infty} \frac{z^{n}}{\Gamma(\eta n+1)}, \\
{ }_{1} K_{1,1}^{(\beta, \eta)_{1}}[1 ; 1,(1, \eta) ; z]=E_{\eta}[z] .
\end{gathered}
$$

Which is the Mittag-Leffler function studied by [7]. 


\subsection{Fractional Claculus of Multiparameter K-Mittag- Leffler Function}

We will prove, the fractional integration and differentation of ${ }_{p} K_{q, k}^{(\beta, \eta)_{m}}[z]$ function with power multipliers into the function of the same form.

Theorem 1. Let $\alpha>0, a \in R, k \in R_{+}=(0, \infty) ; a_{j}, b_{r}, \beta_{i} \in C ; \eta_{i} \in$ $R, \operatorname{Re}\left(\beta_{i}\right)>0 ;(j=1,2, . ., p ; r=1,2, . ., q ; i=1,2, . ., m)$. Then there holds the relation,

$$
\left(I_{0+}^{\alpha}\left[t^{\frac{\beta_{1}}{k}-1}{ }_{p} K_{q, k}^{(\beta, \eta)_{m}}\left[a t^{\frac{\eta_{1}}{k}}\right]\right]\right)(x)=k^{\alpha} x^{\left(\frac{\beta_{1}}{k}+\alpha-1\right)}{ }_{p} K_{q, k}^{(\beta, \eta)_{m}}\left[a x^{\frac{\eta_{1}}{k}}\right] .
$$

Proff. By virtue of (5) and (11), we have

$$
\begin{gathered}
A \equiv\left(I_{0+}^{\alpha}\left[t^{\frac{\beta_{1}}{k}-1} K_{q, k}^{(\beta, \eta)_{m}}\left[a t^{\frac{\eta_{1}}{k}}\right]\right]\right)(x), \\
A \equiv\left(I_{0+}^{\alpha}\left[t^{\frac{\beta_{1}}{k}-1} \sum_{n=0}^{\infty} \frac{\prod_{j=1}^{p}\left(a_{j}\right)_{n, k}\left(a t^{\frac{\eta_{1}}{k}}\right)^{n}}{\prod_{r=1}^{q}\left(b_{r}\right)_{n, k} \prod_{i=1}^{m} \Gamma_{k}\left(\eta_{i} n+\beta_{i}\right)}\right]\right)(x),
\end{gathered}
$$

on interchanging the order of the integration and summation, and using (9), we obtain

$$
A \equiv \sum_{n=0}^{\infty} \frac{\prod_{j=1}^{p}\left(a_{j}\right)_{n, k}}{\prod_{r=1}^{q}\left(b_{r}\right)_{n, k} \prod_{i=1}^{m} \Gamma_{k}\left(\eta_{i} n+\beta_{i}\right)} \frac{\Gamma\left(\frac{n \eta_{1}+\beta_{1}}{k}\right) a^{n} x^{\left(\frac{n \eta_{1}+\beta_{1}}{k}+\alpha-1\right)}}{\Gamma\left(\frac{n \eta_{1}+\beta_{1}}{k}+\alpha\right)},
$$

using (4) and rearranging the terms, we have

$$
\begin{gathered}
A \equiv k^{\alpha} x^{\left(\frac{\beta_{1}}{k}+\alpha-1\right)} \sum_{n=0}^{\infty} \frac{\prod_{j=1}^{p}\left(a_{j}\right)_{n, k}}{\prod_{r=1}^{q}\left(b_{r}\right)_{n, k} \prod_{i=2}^{m} \Gamma_{k}\left(\eta_{i} n+\beta_{i}\right)} \frac{\left(a x^{\frac{\eta_{1}}{k}}\right)^{n}}{\Gamma_{k}\left(n \eta_{1}+\beta_{1}+\alpha k\right)}, \\
A \equiv k^{\alpha} x^{\left(\frac{\beta_{1}}{k}+\alpha-1\right)}{ }_{p} K_{q, k}^{(\beta, \eta)_{m}}\left[a_{1}, . ., a_{p} ; b_{1}, . ., b_{q},\left(\beta_{1}+\alpha k, \eta_{1}\right),\left(\beta_{2}, \eta_{2}\right) . .,\left(\beta_{m}, \eta_{m}\right) ; a x^{\frac{\eta_{1}}{k}}\right],
\end{gathered}
$$

which proves the theorem.

Theorem 2. Let $\alpha>0, a \in R, k \in R_{+}=(0, \infty) ; a_{j}, b_{r}, \beta_{i} \in C ; \eta_{i} \in$ $R, \operatorname{Re}\left(\beta_{i}\right)>0 ;(j=1,2, . ., p ; r=1,2, . ., q ; i=1,2, . ., m)$. Then there holds the relation,

$$
\left(I_{-}^{\alpha}\left[t^{-\alpha-\frac{\beta_{1}}{k}}{ }_{p} K_{q, k}^{(\beta, \eta)_{m}}\left[a t^{-\frac{\eta_{1}}{k}}\right]\right]\right)(x)=k^{\alpha} x^{\left(-\frac{\beta_{1}}{k}\right)}{ }_{p} K_{q, k}^{(\beta, \eta)_{m}}\left[a x^{-\frac{\eta_{1}}{k}}\right] .
$$

Proff. By virtue of (6) and (11), we have

$$
A \equiv\left(I_{-}^{\alpha}\left[t^{-\alpha-\frac{\beta_{1}}{k}}{ }_{p} K_{q, k}^{(\beta, \eta)_{m}}\left[a t^{-\frac{\eta_{1}}{k}}\right]\right]\right)(x),
$$




$$
A \equiv\left(I_{-}^{\alpha}\left[t^{-\alpha-\frac{\beta_{1}}{k}} \sum_{n=0}^{\infty} \frac{\prod_{j=1}^{p}\left(a_{j}\right)_{n, k}\left(a t^{-\frac{\eta_{1}}{k}}\right)^{n}}{\prod_{r=1}^{q}\left(b_{r}\right)_{n, k} \prod_{i=1}^{m} \Gamma_{k}\left(\eta_{i} n+\beta_{i}\right)}\right]\right)(x),
$$

on interchanging the order of the integration and summation, and using (10), we obtain

$$
A \equiv \sum_{n=0}^{\infty} \frac{\prod_{j=1}^{p}\left(a_{j}\right)_{n, k}}{\prod_{r=1}^{q}\left(b_{r}\right)_{n, k} \prod_{i=1}^{m} \Gamma_{k}\left(\eta_{i} n+\beta_{i}\right)} \frac{\Gamma\left(\frac{n \eta_{1}+\beta_{1}}{k}\right) a^{n} x^{-\left(\frac{n \eta_{1}+\beta_{1}}{k}\right)}}{\Gamma\left(\frac{n \eta_{1}+\beta_{1}}{k}+\alpha\right)},
$$

using (4) and rearranging the terms, we have

$$
\begin{gathered}
A \equiv k^{\alpha} x^{\left(-\frac{\beta_{1}}{k}\right)} \sum_{n=0}^{\infty} \frac{\prod_{j=1}^{p}\left(a_{j}\right)_{n, k}}{\prod_{r=1}^{q}\left(b_{r}\right)_{n, k} \prod_{i=2}^{m} \Gamma_{k}\left(\eta_{i} n+\beta_{i}\right)} \frac{\left(a x^{-\frac{\eta_{1}}{k}}\right)^{n}}{\Gamma_{k}\left(n \eta_{1}+\beta_{1}+\alpha k\right)}, \\
A \equiv k^{\alpha} x^{\left(-\frac{\beta_{1}}{k}\right)}{ }_{p} K_{q, k}^{(\beta, \eta)_{m}}\left[a_{1}, . ., a_{p} ; b_{1}, . ., b_{q},\left(\beta_{1}+\alpha k, \eta_{1}\right),\left(\beta_{2}, \eta_{2}\right) . .,\left(\beta_{m}, \eta_{m}\right) ; a x^{-\frac{\eta_{1}}{k}}\right],
\end{gathered}
$$

which proves the theorem.

Theorem 3. Let $\alpha>0, a \in R, k \in R_{+}=(0, \infty) ; a_{j}, b_{r}, \beta_{i} \in C ; \eta_{i} \in$ $R, \operatorname{Re}\left(\beta_{i}\right)>0 ;(j=1,2, . ., p ; r=1,2, . ., q ; i=1,2, . ., m)$. Then there holds the relation,

$$
\left(D_{0+}^{\alpha}\left[t^{\frac{\beta_{1}}{k}-1}{ }_{p} K_{q, k}^{(\beta, \eta)_{m}}\left[a t^{\frac{\eta_{1}}{k}}\right]\right]\right)(x)=k^{-\alpha} x^{\left(\frac{\beta_{1}}{k}-\alpha-1\right)}{ }_{p} K_{q, k}^{(\beta, \eta)_{m}}\left[a x^{\frac{\eta_{1}}{k}}\right] .
$$

Proff. By virtue of (7) and (11), we have

$$
\begin{gathered}
A \equiv\left(D_{0+}^{\alpha}\left[t^{\frac{\beta_{1}}{k}-1} K_{q, k}^{(\beta, \eta)_{m}}\left[a t^{\frac{\eta_{1}}{k}}\right]\right]\right)(x), \\
A \equiv \frac{1}{\Gamma(s-\alpha)}\left(\frac{d}{d x}\right)^{s} \int_{0}^{x} \frac{t^{\frac{\beta_{1}}{k}-1}}{(x-t)^{1+\alpha-s}} \sum_{n=0}^{\infty} \frac{\prod_{j=1}^{p}\left(a_{j}\right)_{n, k}\left(a t^{\frac{\eta_{1}}{k}}\right)^{n}}{\prod_{r=1}^{q}\left(b_{r}\right)_{n, k} \prod_{i=1}^{m} \Gamma_{k}\left(\eta_{i} n+\beta_{i}\right)} d t
\end{gathered}
$$

where $s=[\alpha]+1$

$$
A \equiv \frac{1}{\Gamma(s-\alpha)} \sum_{n=0}^{\infty} \frac{\prod_{j=1}^{p}\left(a_{j}\right)_{n, k} a^{n}}{\prod_{r=1}^{q}\left(b_{r}\right)_{n, k} \prod_{i=1}^{m} \Gamma_{k}\left(\eta_{i} n+\beta_{i}\right)}\left(\frac{d}{d x}\right)^{s} \int_{0}^{x} \frac{t^{\frac{\beta_{1}+n \eta_{1}}{k}-1}}{(x-t)^{1+\alpha-s}} d t
$$

put $t=u x$ the above expression transforms in to the form

$$
A \equiv \frac{1}{\Gamma(s-\alpha)} \sum_{n=0}^{\infty} \frac{\prod_{j=1}^{p}\left(a_{j}\right)_{n, k} a^{n}}{\prod_{r=1}^{q}\left(b_{r}\right)_{n, k} \prod_{i=1}^{m} \Gamma_{k}\left(\eta_{i} n+\beta_{i}\right)}\left(\frac{d}{d x}\right)^{s} x^{\frac{\beta_{1}+n \eta_{1}}{k}+s-\alpha-1}
$$




$$
\times \int_{0}^{1}(1-u)^{s-\alpha-1} u^{\frac{\beta_{1}+n \eta_{1}}{k}-1} d t
$$

using the Beta function formula, we have

$$
A \equiv x^{\frac{\beta_{1}}{k}-\alpha-1} \sum_{n=0}^{\infty} \frac{\prod_{j=1}^{p}\left(a_{j}\right)_{n, k} a^{n}}{\prod_{r=1}^{q}\left(b_{r}\right)_{n, k} \prod_{i=1}^{m} \Gamma_{k}\left(\eta_{i} n+\beta_{i}\right)} \frac{\Gamma\left(\frac{\beta_{1}+n \eta_{1}}{k}\right)}{\Gamma\left(\frac{\beta_{1}+n \eta_{1}}{k}-\alpha\right)} x^{\frac{n \eta_{1}}{k}},
$$

using (4) and rearranging the terms, we obtain

$$
\begin{aligned}
& A \equiv k^{-\alpha} x^{\frac{\beta_{1}}{k}-\alpha-1} \sum_{n=0}^{\infty} \frac{\prod_{j=1}^{p}\left(a_{j}\right)_{n, k}}{\prod_{r=1}^{q}\left(b_{r}\right)_{n, k} \prod_{i=2}^{m} \Gamma_{k}\left(\eta_{i} n+\beta_{i}\right) \Gamma_{k}\left(n \eta_{1}+\beta_{1}-k \alpha\right)}\left(a x^{\frac{\eta_{1}}{k}}\right)^{n}, \\
& A \equiv k^{-\alpha} x^{\frac{\beta_{1}}{k}-\alpha-1}{ }_{p} K_{q, k}^{(\beta, \eta)_{m}}\left[a_{1}, . ., a_{p} ; b_{1}, . ., b_{q},\left(\beta_{1}-\alpha k, \eta_{1}\right),\left(\beta_{2}, \eta_{2}\right) . .,\left(\beta_{m}, \eta_{m}\right) ; a x^{\frac{\eta_{1}}{k}}\right],
\end{aligned}
$$

which proves the theorem.

Theorem 4. Let $\alpha>0, a \in R, k \in R_{+}=(0, \infty) ; a_{j}, b_{r}, \beta_{i} \in C ; \eta_{i} \in$ $R, \operatorname{Re}\left(\beta_{i}\right)>0 ;(j=1,2, . ., p ; r=1,2, . ., q ; i=1,2, . ., m)$. Then there holds the relation,

$$
\left(D_{-}^{\alpha}\left[t^{\alpha-\frac{\beta_{1}}{k}}{ }_{p} K_{q, k}^{(\beta, \eta)_{m}}\left[a t^{-\frac{\eta_{1}}{k}}\right]\right]\right)(x)=k^{-\alpha} x^{\left(-\frac{\beta_{1}}{k}\right)}{ }_{p} K_{q, k}^{(\beta, \eta)_{m}}\left[a x^{-\frac{\eta_{1}}{k}}\right] .
$$

Proff. By virtue of (8) and (11), we have

$$
\begin{gathered}
A \equiv\left(D_{-}^{\alpha}\left[t^{\alpha-\frac{\beta_{1}}{k}}{ }_{p} K_{q, k}^{(\beta, \eta)_{m}}\left[a t^{-\frac{\eta_{1}}{k}}\right]\right]\right)(x), \\
A \equiv \frac{1}{\Gamma(s-\alpha)}\left(-\frac{d}{d x}\right)^{s} \int_{x}^{\infty} \frac{t^{\alpha-\frac{\beta_{1}}{k}}}{(t-x)^{1+\alpha-s}} \sum_{n=0}^{\infty} \frac{\prod_{j=1}^{p}\left(a_{j}\right)_{n, k}\left(a t^{-\frac{\eta_{1}}{k}}\right)^{n}}{\prod_{r=1}^{q}\left(b_{r}\right)_{n, k} \prod_{i=1}^{m} \Gamma_{k}\left(\eta_{i} n+\beta_{i}\right)} d t
\end{gathered}
$$

where $s=[\alpha]+1$

$$
A \equiv \frac{1}{\Gamma(s-\alpha)} \sum_{n=0}^{\infty} \frac{\prod_{j=1}^{p}\left(a_{j}\right)_{n, k} a^{n}}{\prod_{r=1}^{q}\left(b_{r}\right)_{n, k} \prod_{i=1}^{m} \Gamma_{k}\left(\eta_{i} n+\beta_{i}\right)}\left(-\frac{d}{d x}\right)^{s} \int_{x}^{\infty} \frac{t^{\alpha-\frac{\beta_{1}+n \eta_{1}}{k}}}{(t-x)^{1+\alpha-s}} d t
$$

put $t=\frac{x}{u}$ and using the Beta function, the above expression transforms in to the form,

$$
A \equiv x^{-\frac{\beta_{1}}{k}} \sum_{n=0}^{\infty} \frac{\prod_{j=1}^{p}\left(a_{j}\right)_{n, k} a^{n} \Gamma\left(\frac{\beta_{1}+n \eta_{1}}{k}-s\right)(-1)^{s} \Gamma\left(1+s-\frac{\beta_{1}+n \eta_{1}}{k}\right) x^{-\frac{n \eta_{1}}{k}}}{\prod_{r=1}^{q}\left(b_{r}\right)_{n, k} \prod_{i=1}^{m} \Gamma_{k}\left(\eta_{i} n+\beta_{i}\right) \Gamma\left(\frac{\beta_{1}+n \eta_{1}}{k}-\alpha\right) \Gamma\left(1-\frac{\beta_{1}+n \eta_{1}}{k}\right)},
$$


the reflection formula for gamma function, see $([10], 1.60)$,

$$
\frac{1}{\Gamma\left(1-\frac{\beta_{1}+n \eta_{1}}{k}\right)}=\frac{\Gamma\left(\frac{\beta_{1}+n \eta_{1}}{k}\right)}{\Gamma\left(\frac{\beta_{1}+n \eta_{1}}{k}\right) \Gamma\left(1-\frac{\beta_{1}+n \eta_{1}}{k}\right)}=\frac{\Gamma\left(\frac{\beta_{1}+n \eta_{1}}{k}\right) \sin \left[\left(\frac{\beta_{1}+n \eta_{1}}{k}\right) \pi\right]}{\pi},
$$

and

$$
\begin{gathered}
\Gamma\left(\frac{\beta_{1}+n \eta_{1}}{k}-s\right) \Gamma\left(1+s-\frac{\beta_{1}+n \eta_{1}}{k}\right)=\frac{\pi}{\sin \left[\left(\frac{\beta_{1}+n \eta_{1}}{k}-s\right) \pi\right]}, \\
=\frac{\pi}{\sin \left[\left(\frac{\beta_{1}+n \eta_{1}}{k}\right) \pi\right] \cos (s \pi)}=\frac{\pi(-1)^{s}}{\sin \left[\left(\frac{\beta_{1}+n \eta_{1}}{k}\right) \pi\right]},
\end{gathered}
$$

using (17) and (18) in (16), we obtain

$$
A \equiv x^{-\frac{\beta_{1}}{k}} \sum_{n=0}^{\infty} \frac{\prod_{j=1}^{p}\left(a_{j}\right)_{n, k} a^{n} \Gamma\left(\frac{\beta_{1}+n \eta_{1}}{k}\right) x^{-\frac{n \eta_{1}}{k}}}{\prod_{r=1}^{q}\left(b_{r}\right)_{n, k} \prod_{i=1}^{m} \Gamma_{k}\left(\eta_{i} n+\beta_{i}\right) \Gamma\left(\frac{\beta_{1}+n \eta_{1}}{k}-\alpha\right)},
$$

using (4) and rearranging the terms, we obtain

$$
\begin{gathered}
A \equiv k^{-\alpha} x^{-\frac{\beta_{1}}{k}} \sum_{n=0}^{\infty} \frac{\prod_{j=1}^{p}\left(a_{j}\right)_{n, k}}{\prod_{r=1}^{q}\left(b_{r}\right)_{n, k} \prod_{i=2}^{m} \Gamma_{k}\left(\eta_{i} n+\beta_{i}\right) \Gamma_{k}\left(n \eta_{1}+\beta_{1}-k \alpha\right)}\left(a x^{-\frac{\eta_{1}}{k}}\right)^{n}, \\
A \equiv k^{-\alpha} x^{-\frac{\beta_{1}}{k}}{ }_{p} K_{q, k}^{(\beta, \eta)_{m}}\left[a_{1}, . ., a_{p} ; b_{1}, . ., b_{q},\left(\beta_{1}-\alpha k, \eta_{1}\right),\left(\beta_{2}, \eta_{2}\right) . .,\left(\beta_{m}, \eta_{m}\right) ; a x^{-\frac{\eta_{1}}{k}}\right],
\end{gathered}
$$

which proves the theorem.

\subsubsection{Particular Cases}

(A) By putting $k=1$,

(i) in equation (12), then it reduces to the result earlier given by ([3], Theorem 3.1, Equation 6).

(ii) in equation (13), then it reduces to the result earlier given by ([3], Theorem 3.2, Equation 7).

(iii) in equation (14), then it reduces to the result earlier given by ([3], Theorem 3.3, Equation 8).

(iv) in equation (15), then it reduces to the result earlier given by ([3], Theorem 3.4, Equation 9).

(B) By putting $k=1, p=q=m$ and $b_{1}=b_{2}=\ldots=b_{m}=1$,

(i) in equation (12), then it reduces to the result earlier given by ([5], Theorem 4.1, Equation 4.4). 
(ii) in equation (14), then it reduces to the result earlier given by ([5], Theorem 4.2, Equation 4.5).

(C) By putting $k=p=q=1, a_{1}=\rho$ and $b_{1}=1$,

(i) in equation (12), then it reduces to the result earlier given by ([11], Theorem 1, Equation 2.1).

(ii) in equation (13), then it reduces to the result earlier given by ([11],Theorem 3, Equation 2.4).

(iii) in equation (14), then it reduces to the result earlier given by ([11],Theorem 5, Equation 2.6).

(iv) in equation (15), then it reduces to the result earlier given by ([11], Theorem 7, Equation 2.8).

(D) By putting $k=p=q=m=1, a_{1}=\delta$ and $b_{1}=1$,

(i) in (12), then it reduces to the result earlier given by ([12], Theorem 1, Equation 14).

(ii)in (13) then it reduces to the result earlier given by ([12], Theorem 3, Equation 23$)$.

(iii)in (14) then it reduces to the result earlier given by ([12], Theorem 5, Equation 29).

(iv)in (15) then it reduces to the result earlier given by ([12], Theorem 7, Equation 35$)$.

(E) By putting $k=m=1$ and $\beta=1$,

(i) in (12), then it reduces to the result earlier given by ([2], Theorem 2.1, Equation 6).

(ii)in (13) then it reduces to the result earlier given by ([2], Theorem 2.2, Equation 10).

(iii)in (14) then it reduces to the result earlier given by ([2], Theorem 2.3, Equation 14).

(vi)in (15) then it reduces to the result earlier given by ([2], Theorem 2.4, Equation 18).

\section{References}

[1]Dorrego, G.A. and Cerutti, R.A. The K-Mittag-Leffler Function. Int. J. Contemp. Math. Sciences, Vol. 7 (2012) No. 15, 705-716.

[2]Gehlot Kuldeep Singh, Integral Representation and Certain Properties of M- Series Associated with Fractional Calculus. International Mathematical Forum, Vol.8, (2013), no. 9, 415-426. 
[3]Gehlot, Kuldeep Singh and Chena Ram, Integral Representation of KSeries, International Transactions in Mathematical Sciences and computers, Volume 4, No. 2 (2012), pp.387-396.

[4]Gehlot, Kuldeep Singh and Chena Ram, Certain properties of K-Series associated with Fractional calculus, (Communicated for Publication).

[5]Konovska, Paneva Jordanka, Three-Multi-Index Mittag-Leffler Functions, Series and Convergence Theorem, Proceeding of FDA'2012 symposium, Nanjing, China, Paper No. 284, FDA 2012.

[6]Luchko, Yu. F. and Yakubovich, S.B., An operational method for solving some class of introdifferential equations, (Russian). Differ. Uravn., 30, 269280; translation in Differential Equation, 30, 247-256.

[7]Mittag-Leffler, G. Sur la nouvellefonction $E_{\alpha}(z)$. C.RAcad. Sci. Paris 137 (1903) 554-558.

[8]Prabhakar, T.R., A singular integral equation with a generalized MittagLeffler function in the kernal, Yokohama Math. J. 19 (1971), 7-15.

[9]Rafael Diaz and Eddy Pariguan. On hypergeometric functions and Pochhammer k-symbol. Divulgaciones Mathematicas, Vol. 15 No. 2 (2007) 179-192.

[10]Samko, S. G., Kilbas, A.A. and Marichev,O.I. ,Fractional Integral and Derivatives, Theory and Applications. Gordon Breach, Yverdon et al. (1993).

[11]Saxena, R.K., Ram, J. and Suthar, D.L., Fractional Calculus of MittagLeffler functions, Journal of Indian Acad. Math., Vol. 31, No. 1, (2009), pp. 165-172.

[12]Saxena, R.K. and Saigo, M., Certain properties of Fractional Calculus Operator Associated with Generalized Mittag-Leffler function, Frac. Cal. Appl. Anal.,Vol. 8, No. 2, (2005), 141-154.

[13]Sharma, M., Jain, Renu, A note on generalized M-Series as a special function of fractional calculus, Fractional Calculus and applied Analysis, Vol. 12, No.4 (2009), 449-452.

[14]Sharma, M. Fractional Integral and Fractional Differentiation of the M-Series, Fractional Calculus and Applied Analysis, Vol. 11, No. 2 (2008), 
$187-191$.

[15]Virginia S. Kiryakova, Multiple (multiondex) Mittag-Leffler functions and relations to generalized fractional calculus, Journal of Computational and applied Mathematics, 118, (2000) P 241-259.

[16]A. Wiman. Uber den fundamental Satz in der Theories der Funktionen $E_{\alpha}(z)$, Acta Math. 29 (1905) 191-201.

Received: September 15, 2013 